\title{
Rehabilitation outcome evaluation after very severe brain injury
}

\author{
Marlene Reimer and Carole-Lynne LeNavenec \\ Faculty of Nursing, University of Calgary, Calgary, Alberta, Canada
}

Few centres provide long-term therapy for survivors of very severe brain injury who continue in a minimally responsive state. We report on two outcome evaluation projects in association with one such centre in western Canada. In one project a functional scale to detect subtle changes after long-term therapy with the most severely compromised clients (Rancho levels II and III) is being tested. In the other project outcome indicators of change in quality of life after initiation of community-based rehabilitation have been generated by collecting over 400 critical incidents reported by family members, volunteers, staff and a few higher functioning clients. Our intention in this report is to highlight what can be done in terms of rehabilitation and outcome evaluation with clients who seem to be persisting in vegetative or minimally responsive states.

Correspondence should be addressed to: Marlene Reimer, Faculty of Nursing, University of Calgary, Calgary, Alberta, Canada T2N 1N4. Tel: 1403 220-5839, Fax: 1403 284-4803. Email: mareimer@ucalgary.ca

Neither of the research projects described in this paper would have been possible without the support of the Association for the Rehabilitation of the Brain Injured (ARBI) in Calgary, Alberta, Canada. In particular we want to acknowledge the work of Judy Stawnychko as Executive Director and the lead therapists, Mary Anne Ostapovitch, Program Director and Physical Therapist, Brenda Lee-Kemp, Physical Therapist, Sari Martin, Physical Therapist, Sharon Renton, Occupational Therapist, Arlene Lazoruk, Speech and Language Pathologist, Chamine Meghji, Speech and Language Pathologist and Ana Gollega, Occupational Therapist.

We gratefully acknowledge funding from Alberta Community Development and the University of Calgary Faculty of Nursing Research Endowment Fund. Our appreciation also goes to Patrizia Tolle from the University of Bremen, Germany who added conceptual insight in the data analysis of the second project during her study term with us. 
Few centres provide long-term, therapist supervised rehabilitation for adult survivors of very severe brain injury who continue in a state of minimal response to the environment. One such centre is the Association for the Rehabilitation of the Brain Injured (ARBI) in western Canada. In this article we briefly describe that programme and then discuss two research projects to evaluate community-based rehabilitation outcomes with this severely compromised population.

\section{COMMUNITY-BASED REHABILITATION AT ARBI}

This vision for something more, after standard publicly and insurance funded rehabilitation has been exhausted, started with the mother of a brain injured son and her friend. The son that she was told would probably remain in a vegetative state for the rest of his life now lives in his own home with minimal supervision, walks without assistance, writes legibly, speaks intelligibly, and works full-time. Most readers will be able to recount similar recovery stories. What is different about this one is that the two women went on to establish a non-profit organisation that now employs a staff of 30 that includes administrative support, therapists, support workers, and programme leaders, and engages between 60-70 volunteers weekly who contribute in excess of 10,660 hours per year working directly with clients. Most of the 21 clients in the on-site programme are brought in from their place of residence by handi-bus five half-days a week, 11/1/2 months of the year. Another 33 clients in the outreach programme are seen in their own place of residence regularly but less frequently. All of these clients range from Level II to Level V on the Rancho Scale (Malkmus, Booth, \& Kodimer, 1980). Their average length of enrolment in the on-site programme is four years.

Much of the success of this programme has been based on the recruitment and training of dedicated volunteers, most of whom provide three hours or more a week of one-on-one therapy with a particular client. Each client has an individualised programme established and monitored by the therapy team.

\section{OUTCOME EVALUATION}

Like any programme, outcome evaluation is important. Unlike most programmes, measures of functional change and quality of life with sufficient responsiveness for this severely compromised population are essentially unavailable. The existence of the programme with its concentration of clients who would otherwise be considered ineligible for further therapy, and therapists experienced in working with them, has provided a unique opportunity for research. 


\section{DEVELOPMENT OF A SEVERE BRAIN INJURY RECOVERY SCALE}

Approximately half of ARBI's clients are sufficiently functional and responsive to their environment for yearly progress to be measured using existing functional measures such as the JFK Coma Recovery Scale (Giacino \& Kalmar, 1997; Giacino, Kezmarsky, DeLuca, \& Cicerone, 1991), Western Neuro Sensory Stimulation Profile (Ansell \& Keenan, 1989), and the Chedoke-McMaster Stroke Assessment (Gowland et al., 1993). However, the other half are so severely compromised and making such slow, incremental progress that no set of existing measures have been located that are sufficiently sensitive. A team of physiotherapists and occupational and speech language therapists plus the first author, a nurse scientist, are developing and testing a composite of sub-scales to address this need.

The target population is adults who have experienced severe brain injury secondary to trauma, anoxia or cerebral vascular accident, who are clients of ARBI's community-based programme, and who currently function at Level II or III on the Rancho Scale. Responses at these levels range from inconsistent and non-purposeful reaction to stimuli, as evidenced by physiological changes or gross body movements, to vague purposeful responses such as turning the head to sound or occasionally responding to a simple command.

Using modified measurement development methods (Guyatt, Bombardier, $\&$ Tugwell, 1986), the team reviewed the literature and analysed measures currently in use. MEDLINE was searched from 1966 to the present using key words such as brain injury and recovery of function. The therapists already use an extensive battery of measures for which psychometric properties and utility were examined. Information was also sought out on additional approaches. One such approach is the Sensory Modality Assessment Rehabilitation Technique (SMART; Gill-Thwaites, 1997: http://www.rhn.org.uk) which appears to be designed for assessment of clients at a similar level. However, it is required that SMART assessors complete an intensive week of training and completion of an assessment portfolio. Such rigorous training is commendable but not feasible at this time for agencies such as ARBI that have a very limited budget. Thus it was determined that there was a need to proceed with development of a new and potentially complementary measure.

The next step was inductive. Working in pairs, drawing on their extensive professional background, the therapists addressed the question, "What change in one of these clients makes me excited?" The descriptions of those changes were then categorised into items and subscales. In spite of the exhaustive literature review there was often little evidence other than clinical experience for the expected sequence of return of responses to certain stimuli (e.g., olfactory). The variability in sequelae of the initial focal or generalised 
brain insult, associated trauma or deficits affecting other parts of the body, and secondary effects such as spasticity and contractures required creativity in wording and procedures to allow for individual differences.

The items and subscales so compiled were presented for discussion in regular team meetings, revised for clarity and consistency of wording and scoring, and then sent out for review by clinical experts. From early in the development process small components were pre-tested with clients. At the time of writing this comprehensive measure consists of three major subscales, each of which can be used independently or as part of the overall measure. The subscales include responses to environment (e.g., visual, olfactory), motor responses (e.g., head control, trunk control), and communication and swallowing (e.g., yes/no, vocalisation, comprehension). Components of each subscale are further subdivided into items that can be scored on a 7point scale. A score of 1 means no response on all scales. As an example of the fine gradation for each, the first four levels for visual response are $1=$ no response, $2=$ blinks to light or threat; $3=$ fixes gaze $<2$ seconds; $4=$ fixes gaze $>2$ seconds. As a second example vocalisation behaviours can range from $1=$ none, individual does not produce any voice/sound by mouth, not even to pain; to $7=$ vocalises consistently, vocalisations begin to resemble speech sounds (vowels and consonants), are produced spontaneously (e.g., 1-5 instances over 20 minutes) or in response to commands or questions. Items are still being tested and refined but these examples suffice to show just how compromised the target population is, considering that it may take a whole year of volunteer therapy for a client to move up one or two levels. However, clinicians who have worked with this population will also recognise that progress such as being able to maintain head control with support and gaze for $>2$ seconds then enables more active engagement in therapy and improved quality of life for both the client and families.

Once each subscale has been through pre-testing, with further refinements as necessary, the complete measure will be piloted with approximately 40 clients. At that time initial evidence for construct validity, based on expected correlations with similar scales, test-retest and inter-rater reliability will be assessed. Our purpose has been served if this severe brain injury recovery scale allows for quantifying progress to families, health care professionals, funders and donors.

\section{INDICATORS OF CHANGES IN QUALITY OF LIFE}

The purpose of the second project has been to elicit indicators of changes in quality of life. It is commonly assumed that survivors of very severe brain injury have very little quality of life. This assumption arises from conceptual and practical sources. Conceptually, quality of life is often defined as 
requiring awareness of self (Kuhse \& Singer, 1989; Zhan, 1992). Practically, quality of life evaluation in this population is usually based on functional abilities such as self-care and return to work, and proxy estimations by family members or health care providers.

In the project reported here, we set out to explore the quality of life of severely brain injured individuals. We used the critical incident method to elicit observed behaviours and the meanings attributed to them, rather than merely asking for proxy opinion on current quality of life (Flanagan, 1954; 1982). This approach was taken, based on an alternative conceputualisation in which quality of life is defined as the overall state of well-being; physical, psychological and social functioning; and satisfaction with important dimensions of life that cognitively impaired individuals experience, regardless of baseline (Reimer, 1997; 2001; Renwick \& Brown, 1996). For this study baseline was taken as the client's status on admission to the community-based rehabilitation programme, rather than life prior to brain injury.

Using semistructured interviews with 12 clients who were able to comprehend and verbalise in short sentences, 24 family members, 18 volunteers, and 6 therapists (each interviewed about four different clients), over 400 incidents were recorded and transcribed. Using Flanagan's guidelines (1954) which call for a description of a particular incident observed by the person reporting and a judgement by that person as to what was significant about the incident, each participant was asked to describe two positive and two negative incidents which indicated changes in quality of life for a specific client since admission to ARBI. Each interview ended with a request for an incident that indicated, "What makes life good right now?", or (if the response was "nothing"), "What would have to happen to make life good right now?" For example, one mother described how her daughter can now sit propped up in a wheelchair. When asked why she saw that as important her reply was, "Now she can look people in the eye, she can make better eye contact." A higher functioning client described an incident about being able to transfer. What was important to his quality of life was that now "nobody is watching while I take a shower".

Content analysis has revealed six major themes from which emerge indicators of quality of life: behaviour, cognition, communication, emotion/ mood, physical function and capacities, and socio-environmental supports and resources (LeNavenec \& Reimer, 2002). For minimally responsive clients, changes in behaviour such as decreased agitation were seen by family members, volunteers, and staff as improvements. Evidence of enhanced awareness such as visual tracking and the ability to anticipate going outside were seen as affording greater connection with people and satisfaction with self. Changes in non-verbal communication included eye contact and smiling. For example, connecting to the point of being able to smile in response to appropriate stimuli was the culmination of eight 
months of daily therapy for one client. Other indicators under communication included learning to use a communication device and becoming able to indicate approval and disapproval. Achieving head control and reduced spasticity were indicators under physical functioning in that these achievements were seen as important in enhancing visual stimulation. For many clients, receiving one-on-one attention was an important indicator under the social theme. Some of these clients had been receiving little more than custodial care or had come from environments prior to the brain injury in which they had seldom experienced such consistent love and care.

\section{SUMMARY}

By describing this work in progress our intention has been to highlight what can be done in terms of intervention and outcome evaluation with adults who appear to be continuing in a vegetative or minimally conscious state. The significance of these studies is two-fold. First, they have yielded methods for detecting subtle changes in function and quality of life in minimally responsive adults. Second, by demonstrating that such changes can be detected, they have provided evidence that rehabilitation efforts with this population, even long after the initial insult, can make a difference. The broader significance of this report comes in demonstrating the feasibility and effectiveness of a community-based rehabilitation programme, largely delivered by volunteers under therapist supervision, in bringing about small but significant improvements in function and quality of life.

\section{REFERENCES}

Ansell, B. J., \& Keenan, J. E. (1989). The Western Neuro Sensory Stimulation Profile: A tool for assessing slow-to-recover head-injured patients. Archives of Physical Medicine and Rehabilitation, 70, 104-108.

Flanagan, J. C. (1954). The critical incident technique. Psychological Bulletin, 15, 327-358.

Flanagan, J. C. (1982). Measurement of quality of life: Current state of the art. Archives of Physical Medicine and Rehabilitation, 81, 56-59.

Giacino, J. T., \& Kalmar, K. (1997). The vegetative and minimally conscious states: A comparison of clinical features and functional outcome. Journal of Head Trauma Rehabilitation, 12(4), 36-51.

Giacino, J. T., Kezmarsky, M. A., DeLuca, J., \& Cicerone, K. D. (1991). Monitoring rate of recovery to predict outcome in minimally responsive patients. Archives of Physical Medicine and Rehabilitation, 72(11), 897-901.

Gill-Thwaites, H. (1997). The sensory modality assessment rehabilitation technique: A tool for assessment and treatment of patients with severe brain injury in a vegetative state. Brain Injury, 11(10), 724-734. 
Gowland, C., Stratford, P., Ward, M., Moreland, J., Torresin, W., Van Hullenaar, S., Sanford, J., Barreca, S., Vanspall, B., \& Plews, N. (1993). Measuring physical impairment and disability with the Chedoke-McMaster Stroke Assessment. Stroke, 24(1), 58-63.

Guyatt, G. H., Bombardier, C., \& Tugwell, P. X. (1986). Measuring disease-specific quality of life in clinical trials. Canadian Medical Association Journal, 134, 889-895.

Kuhse, H., \& Singer, P. (1989). The quality/quality-of-life distinction and its moral importance for nurses. International Journal of Nursing Studies, 26(3), 203-212.

LeNavenec, C. L., \& Reimer, M. A. (2002). Outcome indicators of quality of life changes for people with traumatic brain injury in a community rehabilitation program. Unpublished data.

Malkmus, D., Booth, B. J., \& Kodimer, C. (1980). Rehabilitation of the head injured adult: Comprehensive cognitive management. Downey CA: Professional Staff Association, Rancho Los Amigos Medical Center.

Reimer, M. A. (1997). Measurement of quality of life in adult onset cognitive impairment. Unpublished doctoral dissertation, University of Calgary, Calgary AB, Canada.

Reimer, M. A. (2001). On being human: Alterations in the sense of being. In C. Stewart-Amidei \& J. A. Kunkel (Eds.), AANN's Neuroscience Nursing: Human responses to neurologic dysfunction (2nd ed., pp. 771-784). Philadelphia: W. B. Saunders.

Renwick, R., \& Brown, I. (1996). The Centre for Health Promotion's conceptual approach to quality of life: Being, belonging, and becoming. In R. Renwick, I. Brown \& M. Nagler (Eds.), Quality of life in health promotion and rehabilitation (pp. 75-86). Thousand Oaks, CA: Sage Publications.

Zhan, L. (1992). Quality of life: Conceptual and measurement issues. Journal of Advanced Nursing, 17, 795-800. 